\title{
Specification, Validation and Run-time Monitoring of SOA Based System-of-Systems Temporal Behaviors
}

\author{
Thomas S. Cook \\ Computer Science Department \\ Naval Postgraduate School \\ Monterey, CA, USA \\ tscoo1@nps.edu
}

\author{
Doron Drusinksy \\ Computer Science Department \\ Naval Postgraduate School \\ Monterey, CA, USA \\ and Time Rover, Inc. \\ Cupertino, CA, USA \\ ddrusins@nps.edu,www.time-rover.com
}

\author{
Man-Tak Shing \\ Computer Science Department \\ Naval Postgraduate School \\ Monterey, CA, USA \\ shing@nps.edu
}

\begin{abstract}
This paper is concerned with the correct specification and validation of temporal behaviors in a Service-Oriented Architecture based system-of-systems. It presents a new formalism, called Message Sequence Chart Assertions (MSC-Assertions), for the specification of global system behaviors, and describes a specification validation technique using scenario simulation based on the JUnit Test Framework. We also describe the armorplating of system-of-systems using runtime execution monitoring of MSC-Assertions.
\end{abstract}

Keywords: Message Sequence Chart (MSC) Assertions, JUnit testing, formal specification, run-time execution monitoring, validation, verification, service-oriented architecture, web services.

\section{Introduction}

Large systems-of-systems (SoSes) are typically made up of a federation of existing systems and developing systems interacting with each other over a network to provide an enhanced capability greater than that of any of the individual systems within the system-of-systems. Serviceoriented architecture (SOA) and the supporting Web Services (WS) technology hold promise to create SoSes that are interoperable, composable, extensible, and dynamically reconfigurable.

A SOA, in the most generic sense, is an architecture that supports the discovery of, binding to, and execution of some resource (service) or composition of resources (services) on a network. Services are applications designed to be reusable, loosely coupled, composable, autonomous, stateless, discoverable, defined by a formal contract, and to conceal underlying logic. As the supporting technology evolves, so too does the concept of a SOA. Today, many large organizations, including the US Department of Defense, are thinking and acting in accordance with the SOA definition found in [10], where SOA is defined as "an architecture that promotes service-orientation through the use of Web Services”.
The increasing use of SOA and WS for complex system-of-systems has raised concerns regarding the correctness and trustworthiness ${ }^{1}$ of the SOA-based SoSes [2]. At the highest abstract level, the functionality of a SOA-based SoS can be viewed as a set of Web Services. A Web Service has two components: a contract defining its external behavior from the clients' point of view, and a business process describing its internal logic via the coordination and composition of other Web Services. The specification of these complex business processes is error prone due to concurrency in activities execution, possibility of communication delay and error, as well as faults in the remote service providers. Often, even if the specifications are correct for an ideal operating environment, they do not operate as well in a less than ideal situation, thus render the resultant SOA-based SoS untrustworthy.

Many researchers proposed to enhance the trustworthiness of the Web Services via formal specification and verification of their business processes [3, 4, 11-14, 16], using light-weight formal methods consisting of the following four steps:

Step 1. Specify the business process in some semi-formal languages (e.g. the Business Processing Execution Language (BPEL));

Step 2. Translate the specifications into formal models (e.g. Linear temporal logic, state machines, Petri nets, process algebras);

Step 3. Specify the desirable functional and non-functional properties of the business process as formal assertions;

Step 4. Verify the formal business process models against the properties using theorem proofing, model checking, or specification-based testing.

1 The term trustworthy software is synonymous with dependable software and hence can be expressed as a composite vector of the software's dependability attributes that include availability, reliability, safety, security (confidentiality and integrity), and maintainability. 
However, for the aforementioned methods to effectively produce trustworthy SoSes, we must address the following concerns:

(1) We need to validate the accuracy and the correctness of the formal-assertion representation of the mission-essential and safety-critical properties of the SoSes. Otherwise, we risk wasting our efforts in verifying the Business Process Models against the wrong set of properties. To address this concern, we present a new formalism and an iterative process for engineers to create and validate system behavior assertions based on Use Case scenarios.

(2) Even if we can correctly capture and specify the system properties, and successfully verify the Business Process models against these properties, there is no guarantee that the SoS will behave as expected during run-time due to possible communication delays and service provider failures. To address this concern, we propose to combine run-time execution monitoring and assertion checking as a means to armor-plate the SOA-based SoSes.

The rest of the paper is organized as follows. Section 2 presents Message Sequence Chart Assertion for specifying distributed system behaviors. Section 3 describes the process for the development and validation of MSC Assertions. Section 4 illustrates armor-plating the SoSes using MSC Assertions and Run-Time Execution Monitoring. Section 5 presents a discussion on the approach and draws the conclusion.

\section{Message Sequence Chart Assertions}

MSC Assertions are a formal-language extension of UML MSC's superimposed with UML statecharts. They have the look and feel of UML MSC's and UML statecharts, yet they are formal and executable. For example, unlike UML MSC's, MSC-Assertions are capable of making a distinction between events that can occur and those that must occur. In addition, MSC Assertions are capable of specifying infinite sets of scenarios.

MSC Assertions are based on Statechart diagrams superimposed on MSC diagrams and augmented with Java (or $\mathrm{C}++$ ) conditions and actions. For example, Figure 1 shows the MSC Assertion for a time-bound requirement of a travel agent service: "R1: The travel agent must obtain bids from at least two airlines and two hotels and return a flight and a hotel matching the customer's request within 30 seconds from the time the customer issues his travel request".

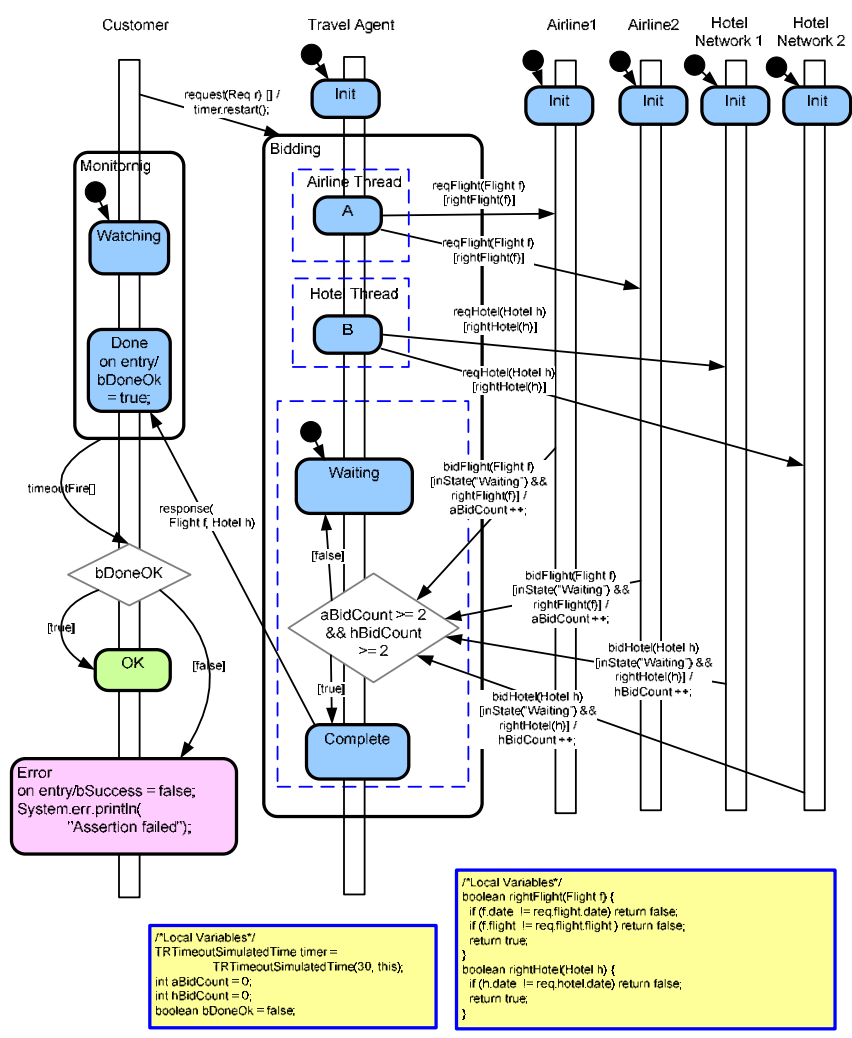

Figure 1. A MSC Assertion for the Travel Agent Service

The MSC-Assertion of Figure 1 looks, for the most part, like a UML MSC, but it enjoys the following unique features:

1. An MSC Assertion is written from the standpoint of an observer, and can be used for run-time monitoring of the target application. Consider for example the message reqFlight(Flight f) sent from the Travel Agent to Airline \#1. While a UML-MSC might consider an interpretation where this event is generated by the Travel Agent, for an MSC Assertion, it is meant that the MSC Assertion should monitor-for, or listen-for, this event flowing from Travel Agent to Airline \#1. Note that while the Travel Agent service may send out many requests for bids, the MSC Assertion only needs to observe two of such requests to satisfy the requirement $\mathrm{R} 1$.

2. An MSC Assertion allows loops and transitions back up the vertical task bar. In Figure 1 for example, the Travel Agent will return to the Waiting state if the condition aBidCount $\geq 2 \& \&$ hBidCount $\geq 2$ is false. This feature is in contrast to UML MSC's where a vertical task bar represents a timeline and where clearly a task cannot move back in time. An MSC Assertion however, considers a vertical task bar as a progression of states, like a state diagram drawn vertically. It therefore permits loops.

3. States and actions. As discussed above and as illustrated in Figure 1, an MSC Assertion task might contain both implicit and explicit states. The purpose 
of explicit states is to specify actions, which are code snippets (written in Java or $\mathrm{C}++$, depending on the code generator chosen) to be performed, such as aBidCount ++ or rightFlight(Flight $h$ ). For example, the Customer will remain in its implicit initial state until the event request(Req $r$ ) is observed leaving the Customer. The Customer then enters the Monitoring state. The Customer will remain in Watching state until either the event response(Flight $f$, Hotel $h$ ) is observed arriving at the Done state, or the timeout event is detected.

4. Java/C ++ underlying language and code generation. An MSC Assertion is a diagrammatic representation of a Java or $\mathrm{C}++$ class that implements the requirement as a monitor. Hence, all variables and functions declared in the local-variables boxes of Figure 1 are actually properties of this generated class.

5. Parameterized events. An MSC Assertion event can contain objects as actual parameters. In Figure 1, the transition annotated with the message bidFlight(Flight f), from Airline \#1 to the Travel Agent, is sent with some Flight object as an argument. Condition guards range over local properties and event arguments (e.g., rightFlight(f) ).

6. An MSC Assertion is an assertion. It uses the same approach described in [6] for assertion statecharts where it announces a success or failure for every witnessed input scenario. It does so using the built-in bSuccess property. The boolean bSuccess is true by default. The developer assigns bSuccess=false as an action wherever s/he wants the assertion to fail. The JUnit test-case then inspects this property to decide whether a particular test-run failed or not.

Figure 1 realized requirement R1 as follows. First note that, in the style of the UML-MSC notation, the assertion contains six tasks, denoted by the six vertical task bars. Also, the assertion contains local variables, timer, aBidCount, hBidCount and bDoneOK, as well as two Boolean functions rightFlight() and rightHotel() for checking the correctness of the itinerary. The MSC Assertion monitoring starts as a request(Req $r$ ) event is observed from the Customer task to the Travel Agent task while the Customer task is in its implicit inital state. The 30 second timer is triggered and the Customer task enters its Watching state. The Customer will remain in Watching state until either the event response(Flight $f$, Hotel $h$ ) from the Travel Agent task (while the latter is in its Complete state) or the timeout event is detected. If the Customer receives the response() message before the timeout event, it will enter the Done state. bDoneOK will be set to true and the subsequent timeout event will cause the Customer task to enter the $O K$ final state. If the Customer task does not receive the response() message before the timeout event, $b D o n e O K$ will remain false and the timeout event will cause the Customer task to enter the Error final state;
bSuccess will be set to false indicating the violation of the requirement.

The Travel Agent task will remain in its Init state until it receives the event request(Req $r$ ), then it will transition to the Bidding state. The Bidding state consists of three concurrent threads, in the style of the UML statechart threads [6]. The Travel Agent task will remain in the Waiting state until it has received at least two airline bids and two hotel bids. It will then transition to the Complete state where the MSC Assertion is ready to observe the event response(Flight $f$, Hotel $h$ ) from the Travel Agent task to the Customer task. Clearly, the Travel Agent task must ensure that the bids received indeed satisfy the customer's request. This constraint is manifested as a condition guard righFlight(f) or rightHotel(h) on the message transition (in other words, MSC Assertion message transitions have the same event[guard]/action look and feel as UML-statechart transitions).

Since we are not interested in the detailed temporal behavior of the Airline tasks and Hotel Network tasks in the requirement $\mathrm{R} 1$, we treat these tasks as black boxes. The MSC Assertion only wants to observe the fact that each of these tasks returns a bid to the Travel Agent task only after they have received a request for bid from the Travel Agent task as follows. Each of these four tasks remains in its Init state until it receives the request for bid message from the Travel Agent task. It then enters its implicit working state. It will transition from its working state to its implicit terminal state when the MSC Assertion observes that the task returns a bid to the Travel Agent task.

\section{Validation of Message Sequence Chart Assertions}

It is important to validate the correctness of the assertions early in the software development process. Unfortunately, users often discover, late in the development process, that their assertions are incorrect and do not work as intended. Possible reasons for incorrect assertions are listed in Table 1 below.

Table 1. Possible reasons for assertion failure

\begin{tabular}{|c|l|}
\hline Case & \multicolumn{1}{|c|}{ Reason } \\
\hline 1. & $\begin{array}{l}\text { Incorrect translation of the natural language } \\
\text { specification to a formal specification. }\end{array}$ \\
\hline 2. & $\begin{array}{l}\text { Incorrect translation of the requirement, as } \\
\text { understood by the modeler, to natural } \\
\text { language. }\end{array}$ \\
\hline 3. & $\begin{array}{l}\text { Incorrect cognitive understanding of the } \\
\text { requirement. This situation typically occurs } \\
\text { when the requirement was driven from the } \\
\text { use case's main success scenario, with } \\
\text { insufficient investigation of other scenarios. }\end{array}$ \\
\hline
\end{tabular}


In [8], we propose the following iterative process for assertion development (Figure 2).

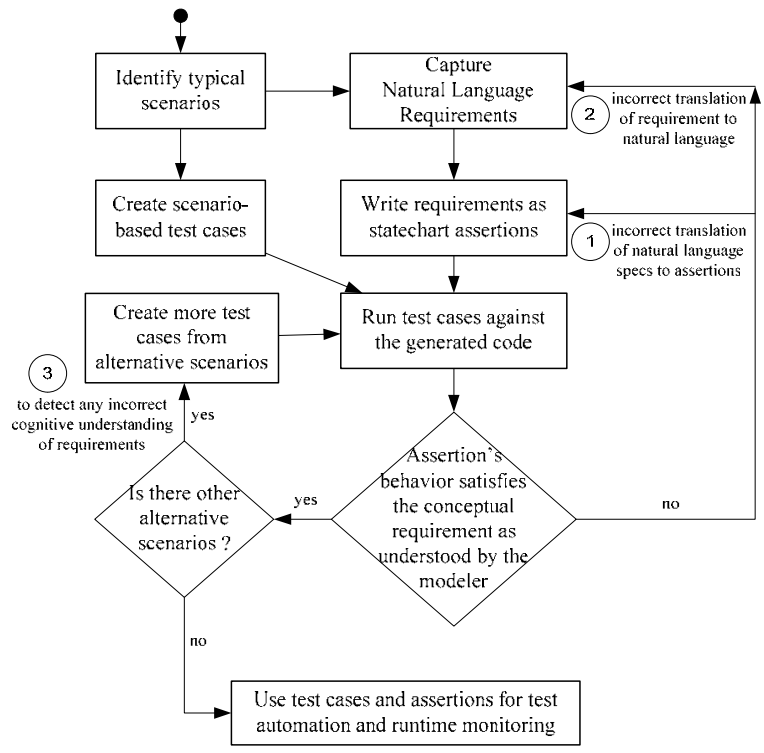

Figure 2. Iterative process for assertion development

Our methodology, as presented in [6-8], is that formal requirements ought to be simulated to assure that the cognitive understanding of the requirement matches the formal specification. To that end, we developed a run-time monitor for MSC Assertions that is fully integrated with the popular JUnit testing framework [1], and created a set of scenarios, using the JUnit testing framework. The scenarios test the MSC Assertion to assure that it announces a success if-and-only if the travel agent returns an acceptable itinerary to the Customer after requesting and obtaining at least two airline bids and at least two hotel bids matching the Customer's request in the prescribed time limit. For example, the following hand-code test case describes a scenario in which the Travel Agent successfully completes its service 25 seconds after receiving the request from the Customer.

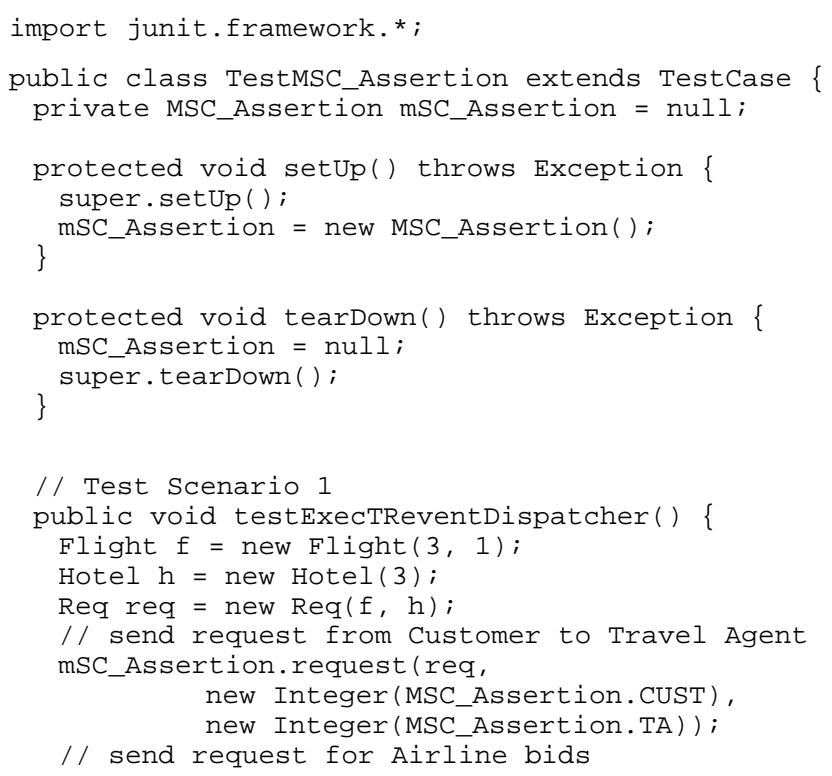

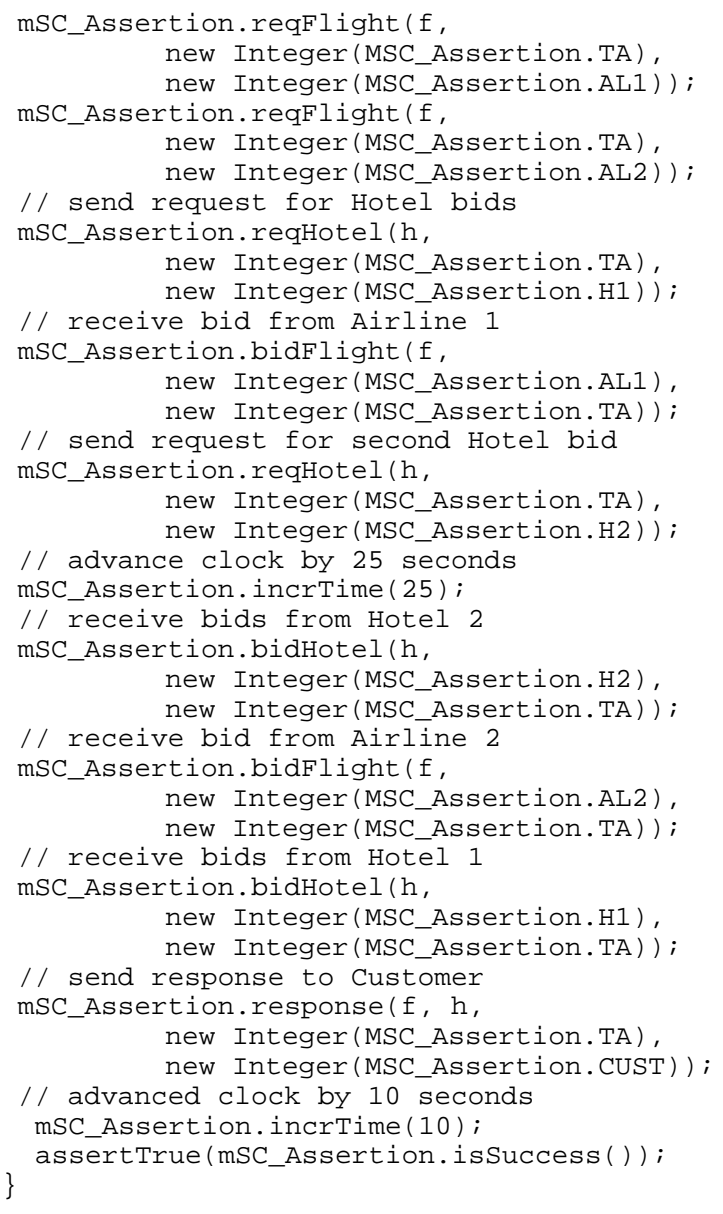

To test the correctness of the assertion, we created two more scenarios by replacing the body of the testExecTReventDispatcher() method with the following code.

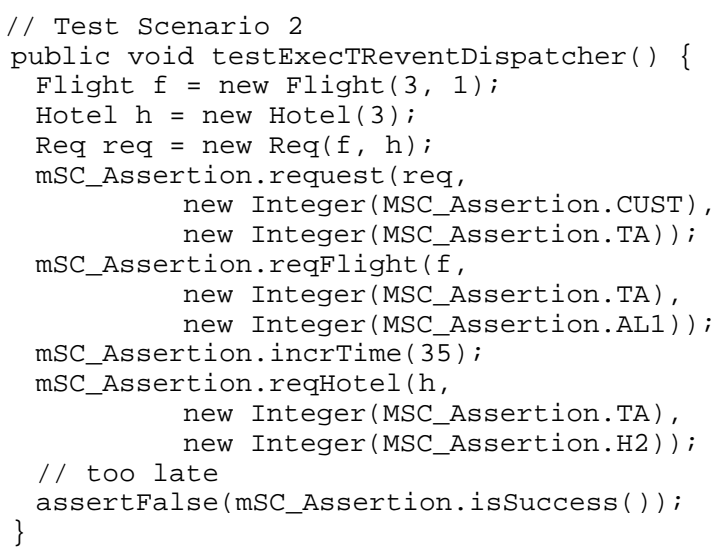




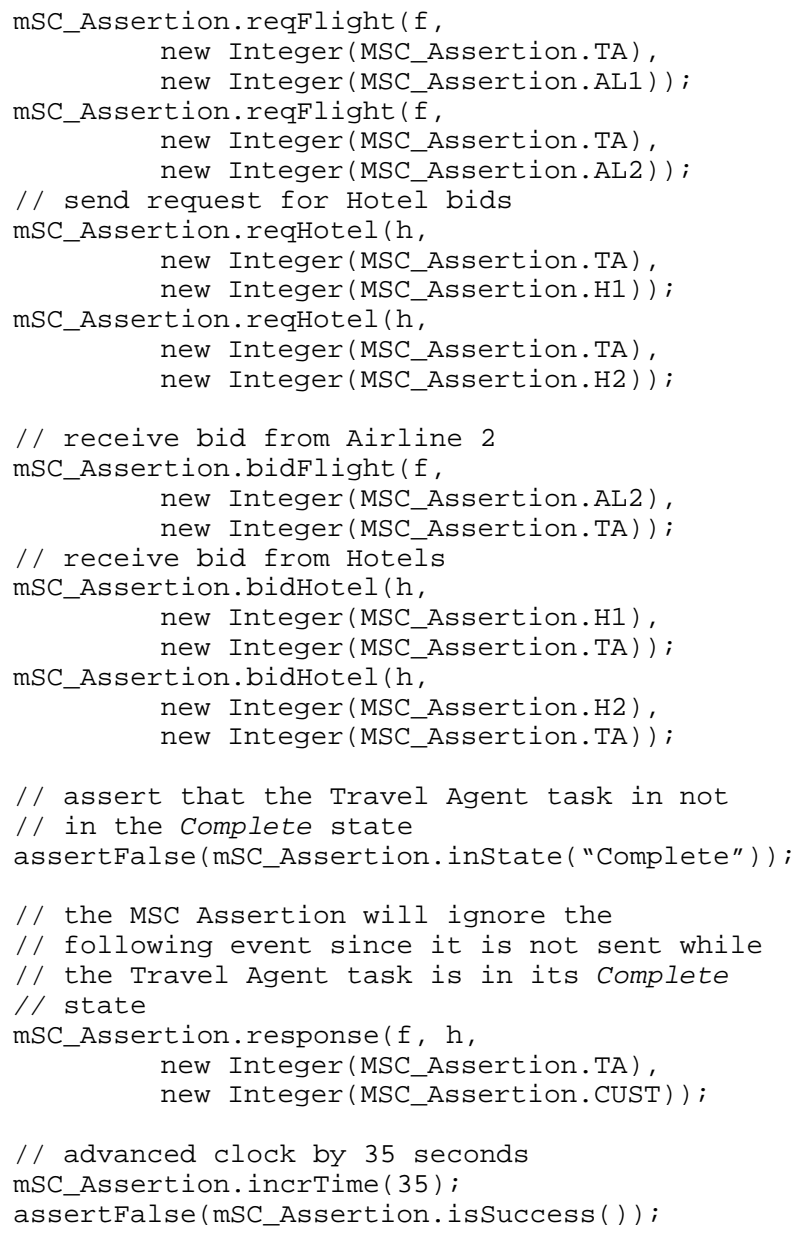

Scenario 2 represents the case where the Travel Agent missed its deadline when it returned the response() back to the Customer. Scenario 3 represents an interesting case where the Travel Agent tried to return a response() back to the Customer without getting at least two Airline bids. Note that the MSC Assertion detects the error and ignores the observed response() event because it was not sent while the Travel Agent task was in its Complete state. The entry action in the Error flowchart box in Figure 1 sets the variable bSuccess to false, which in turn causes mSC_Assertion.isSuccess() to return false and assertFalse() to true.

\section{Run-Time Execution Monitoring of Message Sequence Chart Assertions}

Runtime Execution Monitoring (REM) is a class of methods for tracking the temporal behavior of an underlying application. REM methods range from simple print statement logging methods to run-time tracking of complete formal requirements for verification purposes. NASA used REM to verify the flight code for its Deep Impact project [9]. A recent paper by the authors describes run-time verification of the CARA infusion pump using
UML-statechart models combined with statechart assertions for formal requirement specification [8].

Due to the possibility of communication delay and error, as well as faults in the remote service providers, verification alone can never give us the kind of assurance one expects to have for trustworthy SOA-based SoSes. We need to increase the robustness of these systems by armorplating them against unexpected behaviors. In [5], Drusinsky proposed one form of armor-plating that fortifies the software's exception-handling ability via runtime monitoring of temporal assertions, where formal specifications are translated by a code generator into $\mathrm{C}$, $\mathrm{C}++$, or Java statements to be deployed for catching exceptions in the final product during runtime. Here, we can armor-plate the SoSes by embedding the code generated from the MSC Assertions in the Web Services target code to detect run-time assertion failure. In addition, we can add calls to exception-handlers in the Error flowchart box of the MSC Assertion to enable run-time recovery whenever the MSC Assertion fails during execution of the Web Services.

\section{Discussion and Conclusion}

In this paper, we presented a scenario-based, iterative process and UML-MSC like assertions to help ensure the correctness of formal requirements per the modeler's expectations early in the development process. It is easier for system designers to create and understand MSC Assertions than text-based temporal assertions of the kind found in the literature, such as Linear-time Temporal Logic (LTL) [15] because MSC Assertions are similar to the intuitive and familiar UML MSC's.

Various formal verification techniques suggested in the literature [3, 12-14] approach the correctness of Web Services temporal behaviors by expressing these temporal properties as LTL statements and subsequent model checking. Most model checkers, like SPIN, do not support specifications with real-life constraints such as real-time and time-series. Moreover, LTL has a rather weak expressive power (LTL is sub-regular). In contrast, MSC Assertions use Java/C++ as an underlying language and therefore enjoy Turing-equivalent descriptive power

\section{Acknowledgement}

The research reported in this article was funded in part by a grant from the U.S. Missile Defense Agency. The views and conclusions contained herein are those of the authors and should not be interpreted as necessarily representing the official policies or endorsements, either expressed or implied, of the U.S. Government. The U.S. Government is authorized to reproduce and distribute reprints for Government purposes notwithstanding any copyright annotations thereon. 


\section{References}

[1] K. Beck and E. Gamma, “Test infected: Programmers love writing tests”, Java Report, 3(7) , pp. 37-50, 1998.

[2] K. Birman, R. Hillman and S. Pleisch, "Building network-centric military applications over service oriented architectures”, Proc. SPIE Defense and Security Symposium, pp. 255-266, 29-31 Mar. 2005.

[3] F. van Breugel and M. Koshkina, "Models and Verification of BPEL”, Dept. of Computer Science and Engineering, York University, Toronto, Canada, September 2006. http://www.cse.yorku.ca/ franck/research/drafts/ tutorial.pdf

[4] J. Bhuiyan, S. Nepal and J.Zic, "Checking Conformance Between Business Processes And Web Service Contract In Service Oriented Applications”, Proc. Australian Software Engineering Conference, 18-21 April 2006.

[5] D. Drusinsky, "Specs Can Handle Exceptions”, Embedded Developers Journal, pp. 10-14, Nov. 2001.

[6] D. Drusinsky, Modeling and Verification Using UML Statecharts, Elsevier Publishing, 2006.

[7] D. Drusinsky and M. Shing, Creation and Evaluation of Formal Specifications for System-of-Systems Development., Proc 2005 IEEE International Conference on Systems, Man and Cybernetics, Waikoloa, Hawaii, pp. 1864-1869, Oct 2005.

[8] D. Drusinsky, M. Shing and K. Demir, "Creation and Validation of Embedded Assertions Statecharts", Proc $17^{\text {th }}$ IEEE International Workshop on Rapid Systems Prototyping, Chania, Greece, pp. 17-23, June 2006.

[9] D. Drusinsky and G. Watney, “Applying Run-Time Monitoring to the Deep-Impact Fault Protection Engine”,
Proc $28^{\text {th }}$ IEEE/NASA Software Engineering Workshop, pp. 127-133, Dec 2003.

[10] T. Erl, Service-Oriented Architecture: Concepts, Technology, and Design, Prentice Hall, Upper Saddle River, NJ, 2005.

[11] X. Fu, T. Bultan, and J. Su, "WSAT: A Tool for Formal Analysis of Web Services", Proc. $16^{\text {th }}$ Conf. on Computer Aided Verification, pp. 510-514, 13-17 July 2004.

[12] J. Fisteus, L. Fernández and C. Kloos, “Applying model checking to BPEL4WS business collaborations", Proc. ACM Symposium on Applied Computing, pp. 826830, 13-17 Mar. 2005.

[13] J. Garcia-Fanjul, J. Tuya, and de la Riva, "Generating Test Cases Specifications for BPEL Compositions of Web Services Using SPIN", Proc. Int, Workshop on Web Services Modeling and Testing (WS-MaTe 2006), 9 June 2006.

[14] H. Huang and R. Mason, "Model checking technologies for Web services”, Proc 2nd Int. Workshop on Collaborative Computing, Integration, and Assurance and $4^{\text {th }}$ IEEE Workshop on Software Technologies for Future Embedded and Ubiquitous Systems, 27-28 April 2006.

[15] A. Pnueli, “The Temporal Logic of Programs”, Proc. $18^{\text {th }}$ IEEE Symp. on Foundations of Computer Science, pp. 46-57, 1977.

[16] W. Tsai, X. Wei, Y. Chen and R. Paul, “A robust testing framework for verifying Web services by completeness and consistency analysis”, Proc. 2005 IEEE International Workshop on Service-Oriented System Engineering (SOSE'05), pp. 151-158, 20-21 Oct. 2005. 